\title{
Effect of Silver Nanoparticles on Biofilm Producing Multi Drug Resistant Uro-pathogenic E. coli isolated from Catheterized Patients in Mansoura University Hospital
}

\author{
${ }^{1}$ Eman Nashat*, ${ }^{1}$ Magda M. El Nagdy, ${ }^{2}$ Ghada F. Weefky, ${ }^{1}$ Yasmin Nabiel \\ ${ }^{1}$ Department of Medical Microbiology and Immunology, Faculty of Medicine, Mansoura University, Mansoura, Egypt \\ ${ }^{2}$ Department of Anesthesia and Surgical ICU, Faculty of medicine, Mansoura university, Mansoura, Egypt
}

Key words:
biofilm; CAUTI; MDR
UPEC; silver nanoparticles

*Corresponding Author:
Eman Nashat
Department of Medical
Microbiology \&Immunology,
Faculty of Medicine, Mansoura
University, Egypt.
020-1210724748
emannashat91@ gmail.com

ABSTRACT

Background: Uro-pathogenic E. coli (UPEC) is considered one of the members of the extra-intestinal pathogens, that predominantly cause urinary tract infections. Objectives: Assessment of the prevalence of MDR UPEC in catheterized patients admitted into Mansoura University Hospital, then detection of biofilm production by isolated MDR UPEC strains, and finally testing the effect of silver nanoparticles on biofilm producing ability of the isolated strains. Methodology: 155 urine samples were processed to detect E. coli strains, Antibiotic susceptibility profile of resulting E. coli isolates was determined by Kirby-Bauer disc diffusion method to select MDR UPEC strains, then detection of biofilm production using tissue culture plate method among isolated MDR UPEC strains, and finally evaluation of anti-biofilm activity of silver nanoparticles (AgNPs) by re-detection of biofilm formation after incubation with various concentrations of silver nanoparticles. Results: Out of these urine samples, 35 isolates $(22.6 \%)$ revealed E. coli. Out of them, 34 isolates (97.1\%) were MDR E. coli. 29 isolates (85\%) of MDR UPEC were biofilm producers. The antibiofilm effect was observed in dose dependent manner. Silver nanoparticles with concentration of $100 \mu \mathrm{g} / \mathrm{ml}$, recorded maximum antibiofilm effect (63.22\%) followed by concentrations of 75, 50, and $25 \mu \mathrm{g} / \mathrm{ml}$ which were able to eliminate the biofilm formation of MDR UPEC on the plate surface by (59.91\%), (55.24\%), (43.61\%) respectively. Conclusion: Silver nanoparticles play a major role in the inhibition of biofilm formation by MDR UPEC.

Abbreviations: (AgNPs, Silver nanoparticles; CAUTI, Catheter associated urinary tract infection; MDR, Multidrug resistance; TCP, Tissue culture plate; TSB, Trypticase soy broth; UPEC, Uropathogenic E. coli).

\section{INTRODUCTION}

Uro-pathogenic E. coli (UPEC) is considered one of the members of the extra-intestinal pathogens, that predominantly cause urinary tract infections ${ }^{\mathbf{1}}$.

Approximately $12 \%$ to $16 \%$ of adult hospitalized patients are supposed to have an indwelling urinary catheter at some time during their hospitalization, and each day the indwelling urinary catheter remains, a patient has a $3 \%$ to $7 \%$ increased risk of acquiring a catheter-associated urinary tract infection (CAUTI) ${ }^{2,3}$.

Catheter-associated urinary tract infection can lead to a wide variety of complications that can lead to prolonged hospital stay, and increased cost with higher morbidity and mortality ${ }^{4}$. Multi-drug resistant E. coli have a variety of virulence factors that allow them to establish an infection, including adhesins, toxins, host defense avoidance mechanisms and iron acquisition systems $\mathbf{5}$.

Biofilms are communities of microorganisms attached to a firm surface. These adherent cells regularly embedded within a self-produced matrix of extracellular polymeric substance ${ }^{6}$. The major problem is that biofilms are very resistant to host defense mechanisms and antibiotic treatment, compared to their planktonic cells ${ }^{7}$.

Nano scale materials have appeared as one of the most promising strategies to control biofilm infections related to indwelling medical devices, especially due to their high surface area to volume ratio and unique chemical and physical properties ${ }^{\mathbf{8}}$. The ability of AgNPs to block bacterial growth, and to prevent the glycocalyx formation, was helpful to prevent bacterial adhesion and following the biofilm formed on the medical devices ${ }^{\mathbf{9 , 1 0}}$.

The study allowed to estimate the prevalence of MDR UPEC in catheterized patients admitted into Mansoura University Hospital, then detect biofilm production by isolated MDR UPEC strains, and finally testing the effect of silver nanoparticles on biofilm producing ability of the isolated strains. 


\section{METHODOLOGY}

\section{Study population:}

The study was carried out on 155 urine samples obtained from catheterized patients admitted to Mansoura University Hospital showing evidence of CAUTI. The study was conducted in Medical Microbiology and immunology Department, Faculty of medicine, Mansoura University, during the period from November 2019 to November 2020. The protocol of this study was accepted by Institutional Review Board (IRB), Faculty of Medicine, Mansoura University; code number: MS.19.09.783.

The following data were collected from the patients and included: name, age, gender, admission place, associated comorbidities (as diabetes, hypertension, chronic kidney disease, congestive heart failure and others).

\section{Collection and processing of samples:}

One hundred fifty-five non repetitive clinical specimens of urine were subjected to culture on CLED followed by standard biochemical tests to isolate $E$. coli then antibiotic susceptibility testing was carried out by using the following antibiotics (Oxoid, UK): (AK, Amikacin; AMC, Amoxicillin/clavulanate; ATM, Aztreonam, CTX, cefotaxime; CEC, cefaclor; CN, Gentamicin; FEP, Cefepime; NOR, norfloxacin; MEM, Meropenem; F, Nitrofurantoin; TE, tetracycline; SXT, Trimethoprim/ sulfamethoxazole; CIP, ciprofloxacin; TPZ, Piperacillin / Tazobactam), based on the recommendations of the Clinical and Laboratory Standards Institute (CLSI 2018).

\section{Detection of biofilm formation by Tissue Culture Plate method: 11,12}

Each MDR E. coli isolate was inoculated in $10 \mathrm{~mL}$ of fresh TSB broth containing $1 \%$ glucose and incubated at $37^{\circ} \mathrm{C}$ for 24 hours. The cultures were then diluted (1:100) using fresh medium. Each well of the TCP was loaded with $0.2 \mathrm{~mL}$ of the diluted bacterial culture. Only sterile broth was served as blank (negative control). Reference strain (E. coli ATCC 25922 strain used as a positive control, (kindly offered from Naval Medical Research Unit-3 (NAMRU-3), Cairo, Egypt) was also diluted and incubated.

The plates were incubated at $37^{\circ} \mathrm{C}$ for 24 hours. After incubation, culture medium in the each well was removed by gentle tapping. Floating bacteria were removed by washing the plate twice with $0.2 \mathrm{~mL}$ PBS $(\mathrm{pH}$ 7.2) and the plates were allowed to dry in an inverted position at room temperature.

The adherent biofilms formed by isolates were fixed by $2 \%$ sodium acetate then washed, well dried, and stained with $0.1 \%$ crystal violet (aqueous) for 10 minutes at room temperature. Excess stain was removed by washing again with PBS and allowed to air dry at room temperature. Then $200 \mu \mathrm{L}$ of $95 \%$ ethanol was added to stained wells and dye was allowed to solubilize by incubating for 15 minutes at room temperature. The optical density (OD) of stained adherent biofilm was measured by plate reader at $590 \mathrm{~nm}$ wavelength. Each assay was performed in triplicate and the mean values of crystal violet absorbance \pm SD

Evaluation of anti-biofilm effect of silver nanoparticles: ${ }^{13}$

A stock solution of water-soluble silver nanoparticles (spherical) was purchased from Nano Tech, Egypt. Each MDR biofilm producer E. coli isolate was inoculated in $10 \mathrm{~mL}$ of fresh TSB broth containing $1 \%$ glucose and incubated at $37^{\circ} \mathrm{C}$ for 24 hours. The cultures were then diluted $(1: 100)$ using fresh medium. Each well of the TCP was loaded with $0.1 \mathrm{~mL}$ of the diluted bacterial culture.

Different concentrations of silver nanoparticles were prepared from stock $200 \mu \mathrm{g} / \mathrm{ml}$, then $0.1 \mathrm{ml}$ of these dilutions were added to the wells as the following: First column of wells was loaded with silver nanoparticles with concentration $200 \mu \mathrm{g} / \mathrm{ml}$ to reach the desired concentration $(100 \mu \mathrm{g} / \mathrm{ml})$, second, third, and fourth columns, AgNPs were loaded with concentrations 150, $100,50 \mu \mathrm{g} / \mathrm{ml}$ to reach $(75,50,25 \mu \mathrm{g} / \mathrm{ml})$ respectively, fifth column was served as negative control (untreated biofilm), then the plate was incubated at $37^{\circ} \mathrm{C}$ for overnight. After incubation, gentle tapping of the plates was done. The wells were washed with $0.2 \mathrm{ml}$ of phosphate buffer saline ( $\mathrm{pH}$ 7.2) four times and stained with $0.1 \%$ crystal violet for $30 \mathrm{~min}$. Excess stain was washed with distilled water and plates were dried properly. Optical densities (OD) of stained adherent biofilm were obtained with a micro-ELISA auto reader at wavelength $590 \mathrm{~nm}$.

The percentage inhibition of biofilm activity was calculated using the following equation (Abdel Rahim and Mohamed $\left.{ }^{14}\right)$ : Biofilm inhibition percentage $(\%)=$ 1 - (absorbance of cells treated with Ag NPs / absorbance of non-treated wells) $\times 100$

\section{Statistical analysis:}

Data were analyzed using the Statistical Package of Social Science (SPSS) program for Windows (Standard version 21). The normality of data was first tested with one-sample Kolmogorov-Smirnov test. Qualitative data were described using number and percent. Continuous variables were presented as mean $\pm \mathrm{SD}$ (standard deviation) for normally distributed data. Paired t test was used to compare quantitative parametric data at two different concentrations at the same group. The threshold of significance is fixed at 5\% level.The results was considered significant when $\mathrm{p} \leq 0.05$. The smaller the p-value obtained, the more significant were the results. 


\section{RESULTS}

Our study was carried out on 155 urine samples obtained from catheterized patients admitted to Mansoura University Hospital showing evidence of CAUTI. The mean age of study subjects was 72 years. CAUTI was more among males $(80.6 \%)$ than females $(19.4 \%) .(80.6 \%)$ of CAUTI were admitted to different hospital wards whereas $(19.4 \%)$ were admitted to ICU. A correlation between CAUTI and co-morbidities as diabetes mellitus (40.0\%), hypertension (27.7\%), Congestive heart failure $(3.9 \%)$ as these co-morbidities can aggressively affect the immune system.
As regard the distribution of growth among the studied group, this study showed that 35 isolates $(22.6 \%)$ were E. coli. On testing antibiotic susceptibility by disk diffusion method to detect MDR UPEC, cefaclor showed the highest resistance $(91.4 \%)$ followed by amoxicillin/clavulanate\& trimethoprim/ sulfamethoxazole (88.6\%), norfloxacin (82.9\%), ciprofloxacin\& tetracycline $(80 \%)$, cefotaxime $(77.1 \%)$, aztreonam (68.6\%), cefepime (54.3\%). Amikacin \& piperacillin-tazobactam \& meropenem $(5.7 \%)$ showed the least resistance followed by nitrofurantoin $(29.4 \%)$ and gentamicin $(37.1 \%)$ as showed in table 1.

Table1: Antibiotic resistance among the studied uro-pathogenic $E$. coli group $(n=35)$ :

\begin{tabular}{|l|c|c|c|}
\hline \multicolumn{1}{|c|}{ Antibiotics resistance } & Resistant & Sensitive & Intermediate resistant \\
\hline Amoxicillin /clavulanate (AMC) & $31(88.6 \%)$ & $3(8.6 \%)$ & $1(2.9 \%)$ \\
\hline Piperacillin/tazobactam (TPZ) & $2(5.7 \%)$ & $26(74.3 \%)$ & $7(20.0 \%)$ \\
\hline Cefepime (FEP) & $19(54.3 \%)$ & $12(34.3 \%)$ & $4(11.4 \%)$ \\
\hline Cefotaxime (CTX) & $27(77.1 \%)$ & $8(22.9 \%)$ & $0(0 \%)$ \\
\hline Cefaclor (CEC) & $32(91.4 \%)$ & $1(2.9 \%)$ & $2(5.7 \%)$ \\
\hline Aztreonam (ATM) & $24(68.6 \%)$ & $10(28.6 \%)$ & $1(2.9 \%)$ \\
\hline Meropenem (MEM) & $2(5.7 \%)$ & $33(94.3 \%)$ & $0(0 \%)$ \\
\hline Gentamicin (CN) & $13(37.1 \%)$ & $21(60.0 \%)$ & $1(2.9 \%)$ \\
\hline Amikacin (AK) & $2(5.7 \%)$ & $30(85.7 \%)$ & $3(8.6 \%)$ \\
\hline Tetracycline (TE) & $28(80.0 \%)$ & $7(20.0 \%)$ & $0(0 \%)$ \\
\hline Norfloxacin (NOR) & $29(82.9 \%)$ & $5(14.3 \%)$ & $1(2.9 \%)$ \\
\hline Ciprofloxacin (CIP) & $28(80.0 \%)$ & $6(17.1 \%)$ & $1(2.9 \%)$ \\
\hline Trimethoprim /sulfamethoxazole (SXT) & $31(88.6 \%)$ & $4(11.4 \%)$ & $0(0 \%)$ \\
\hline Nitrofurantoin (F) & $10(29.4 \%)$ & $22(64.7 \%)$ & $2(5.9 \%)$ \\
\hline
\end{tabular}

According to the definition of MDR E. coli as acquired non-susceptibility to at least one agent in three or more antimicrobial categories, the percentage of MDR E. coli among the studied uro-pathogenic E. coli was $(97.1 \%)$.

In our study, (85\%) of MDR UPEC isolates were biofilm producers. The current study revealed that biofilm producers were distributed in three categories; weak $(11.7 \%)$, moderate $(64.7 \%)$ and strong $(8.8 \%)$ as shown in table 2 .

Table 2: Detection of biofilm production of MDR UPEC by tissue culture plate method:

\begin{tabular}{|c|c|}
\hline Biofilm production & MDR $\boldsymbol{E}$. coli $(\mathbf{n}=\mathbf{3 4})$ \\
\hline Non producer & $5(14.7 \%)$ \\
Weak & $4(11.7 \%)$ \\
Moderate & $22(64.7 \%)$ \\
Strong & $3(8.8 \%)$ \\
\hline
\end{tabular}

In the current study, the antibiofilm effect of AgNPs was observed as dose dependent manner; silver nanoparticles with concentration of $100 \mu \mathrm{g} / \mathrm{ml}$, recorded maximum antibiofilm effect $(63.22 \%)$ followed by concentrations of 75,50 , and $25 \mu \mathrm{g} / \mathrm{ml}$ which were able to eliminate the biofilm formation of MDR UPEC on the plate surface by $(59.91 \%),(55.24 \%),(43.61 \%)$ respectively as shown in table 3 .

Table 3: Inhibition percentage of biofilm production observed by different concentrations of silver nanoparticles $(100,75,50,25 \mu \mathrm{g} / \mathrm{ml})$ :

\begin{tabular}{|c|c|c|c|}
\hline $\begin{array}{c}\text { Silver } \\
\text { nanoparticle } \\
\text { concentration }\end{array}$ & $\begin{array}{c}\text { Biofilm } \\
\text { inhibition } \\
\text { percentage }\end{array}$ & Paired t test & P value \\
\hline $100 \mu \mathrm{g} / \mathrm{ml}$ & $63.22 \pm 9.26$ & conc 100 vs. conc 75 & $\leq 0.001^{*}$ \\
\hline $75 \mu \mathrm{g} / \mathrm{ml}$ & $59.91 \pm 10.18$ & conc 100 vs. conc 50 & $\leq 0.001^{*}$ \\
\hline $50 \mu \mathrm{g} / \mathrm{ml}$ & $55.24 \pm 17.16$ & conc 100 vs. conc 25 & $\leq 0.001^{*}$ \\
\hline $25 \mu \mathrm{g} / \mathrm{ml}$ & $43.61 \pm 20.63$ & conc 75 vs. conc 50 & $0.009^{*}$ \\
\hline- & - & conc 75 vs. conc 25 & $\leq 0.001^{*}$ \\
\hline- & - & conc 50 vs. conc 25 & $\leq 0.001^{*}$ \\
\hline
\end{tabular}




\section{DISCUSSION}

The mean age of the study subjects was 62 years compared with 64 years in a study done by Niveditha et al, ${ }^{15}$. We found that CAUTI was most frequent in the age group (60-88 years) as it represented by $(65.5 \%)$ and this result agreed with Letica-Kriegel et al, ${ }^{16}$ who reported a percentage $(49.9 \%)$. This might be attributed to suffering from obstructive uropathies like benign prostatic hyperplasia which lead to urinary retention and high post-void residue and those are considered risk factors for infection in this elderly age.

This study showed that CAUTI was more among males $(80.6 \%)$ than females $(19.4 \%)$. This finding was similar to that reported by Sabir et al, ${ }^{17}$ as they declared that the prevalence in male patients was $(78.5 \%)$ and in female was $(21.5 \%)$. The difference could be due to the high prevalence of benign prostatic hyperplasia which is an indication for catheter insertion in males.

$80.6 \%$ of CAUTI were admitted to different hospital wards whereas $19.4 \%$ were admitted to ICU, Lewis et al, ${ }^{18}$ reported similar findings as they announced that $(72 \%)$ occurred in non-ICU patients, and (28\%) occurred in ICU patients. This could be explained by different levels of infection surveillance and prevention efforts as they targeted the ICU patients more than nonICU patients.

A correlation between CAUTI and co-morbidities as diabetes mellitus (40.0\%), hypertension (27.7\%), Congestive heart failure $(3.9 \%)$. This goes with those reported by Niveditha et al, ${ }^{15}$ as they found that the most common underlying illness was diabetes mellitus, which was found in $44 \%$ of the patients, hypertension in $36 \%$ patients, followed by congestive heart failure in $10 \%$ patients. Such co-morbidities can aggressively affect the immune system. In addition, chronic kidney disease was also associated with CAUTI, and this finding was like those reported by Hsiao et al, ${ }^{\mathbf{1 9}}$, this association is mostly due to metabolic abnormalities resulting in alterations in primary host defense mechanisms so chronic kidney disease patients more prone to infection.

For the distribution of growth among the studied group, this study showed that 35 isolates $(22.6 \%)$ were $E$. coli isolates. Among the studied $35 E$. coli isolates, the highest resistance was to cefaclor $(91.4 \%)$ followed by amoxicillin/clavulanate \& trimethoprim/ sulfamethoxazole (88.6\%), norfloxacin (82.9\%), ciprofloxacin\& tetracycline $(80 \%)$, cefotaxime $(77.1 \%)$, aztreonam $(68.6 \%)$, cefepime $(54.3 \%)$, and the least resistance was to amikacin $\&$ piperacillin-tazobactam $\&$ meropenem $(5.7 \%)$ followed by nitrofurantoin $(29.4 \%)$ and gentamicin $(37.1 \%)$. This pattern of antibiotic resistance among UPEC isolates was almost like that stated by Tabasi et $\mathrm{al}^{\mathbf{2 0}}{ }^{\text {, }}$ where the isolates studied showed the least resistance to antibiotics such as meropenem $(0 \%)$, amikacin $(3.6 \%)$, and $(5.1 \%)$ in each of piperacillin/tazobactam and nitrofurantoin. This may be due to misuse of these antibiotics, self-medication, and development of resistant isolates that are transmitted from one patient to another ${ }^{21}$.

According to definition of MDR E. coli as acquired non-susceptibility to at least one agent in three or more antimicrobial categories, the percentage of MDR E. coli among the studied uro-pathogenic $E$. coli in this study was $(97.1 \%)$. Similar findings were documented by Elsayed Gawad et al, ${ }^{22}$ as their research revealed a high incidence of MDR phenotype (90.85\%) among UPEC isolates.

Bacterial biofilm has long been considered as a virulence factor contributing to infection associated with various medical devices. In our study, detection of biofilm formation of clinical isolates was carried out by microtitre plate method which is the gold standard method for biofilm detection ${ }^{23}$.

In the present study, $85 \%$ of MDR UPEC isolates were biofilm producers. This follows what was documented by Elsayed Gawad et al, ${ }^{\mathbf{2 2}}$ as their research revealed a significant correlation between biofilm production and MDR UPEC represented by (81.13\%). Previous studies indicated a strong correlation between biofilm production and resistance to multiple antibiotics. High level of biofilm production might be because catheter represented an excellent medium for biofilm formation and this study was conducted only in catheterized patients.

The current study revealed that biofilm producers were distributed in three categories weak (11.7\%), moderate $(64.7 \%)$ and strong $(8.8 \%)$. whereas the study of Maharjan et al, ${ }^{24}$ showed that biofilm producers were $(21 \%, 14 \%$, and $11 \%)$ weak, moderate, and strong biofilm producers respectively. This might be due to $E$. coli forms biofilms on static assays in a methoddependent fashion, depending on strain, and it is strongly modulated by culture conditions.

In the recent years, various approaches have been developed for combating biofilms. Nanotechnology provides a useful approach in biofilm control ${ }^{25}$. In the current study, the inhibition of biofilm formation of MDR UPEC by silver nanoparticles was done by microtiter plate assay using different concentrations, this clearly revealed that all the tested concentrations inhibited biofilm of MDR UPEC. Results were represented as inhibition percentage of biofilm development. The antibiofilm effect was observed as dose dependent manner. Silver nanoparticles with concentration of $100 \mu \mathrm{g} / \mathrm{ml}$, recorded maximum antibiofilm effect $(63.22 \%)$ followed by concentrations of 75,50 , and $25 \mu \mathrm{g} / \mathrm{ml}$ which were able to eliminate the biofilm formation of MDR UPEC on the plate surface by $(59.91 \%),(55.24 \%),(43.61 \%)$ respectively.

Similar results were also reported by Namasivayam et al, ${ }^{13}$ against $E$. coli. They found that $100 \mu \mathrm{g} / \mathrm{ml}$ of AgNPs resulted in $80 \%$ reduction in biofilm, $75 \mu \mathrm{g} / \mathrm{ml}$ 
of AgNPs resulted in about $70.5 \%$ reduction in biofilm formation, $50 \mu \mathrm{g} / \mathrm{ml}$ of $\mathrm{AgNPs}$ resulted in $65 \%$ reduction in biofilm and $25 \mu \mathrm{g} / \mathrm{ml}$ of AgNPs resulted in about $54.4 \%$ reduction in biofilm formation. Our results are slightly different than those mentioned, this might be explained by the fact that we carried out our evaluation over MDR E. coli. Further research and development are necessary to translate this technology into therapeutic and preventive strategies.

\section{CONCLUSION}

Silver nanoparticles play a major role in the inhibition of biofilm formation by MDR UPEC and on turn makes its treatment by antibiotics much easier.

This manuscript has not been previously published and is not under consideration in the same or substantially similar form in any other reviewed media. I have contributed sufficiently to the project to be included as author. To the best of my knowledge, no conflict of interest, financial or others exist. All authors have participated in the concept and design, analysis, and interpretation of data, drafting and revising of the manuscript, and that they have approved the manuscript as submitted.

\section{REFERENCES}

1. Struelens MJ, Denis O\& Rodriguez-Villalobos H: Microbiology of nosocomial infections: progress and challenges. Microbes Infect. 2004;6(11):10438.

2. McGuckin M: The patient survival guide: 8 simple solutions to prevent hospital and healthcareassociated infections. New York,NY. Demos Medical Publishing. 2012.

3. Lo E, Nicolle LE, Coffin SE, Gould C, Maragakis LL, Meddings $\mathrm{J}$, et al: Strategies to prevent catheter-associated urinary tract infections in acute care hospitals: update. Infection Control and Hospital Epidemiology. 2014;35:464-79.

4. Bagchi I, Jaitly NK, Thombare VR. Microbiological Evaluation of Catheter Associated Urinary Tract Infection in a Tertiary Care Hospital. PJSR. 2015;8(2):23-29.

5. Kaper JB, Nataro JP, Mobley HLT: "Pathogenic Escherichia coli," Nature Reviews Microbiology. 2004, 123-140. doi: 10.1038/nrmicro818.

6. Flemming $\mathrm{H}$, Wingender $\mathrm{J}$ : The biofilm matrix. Nat Rev Microbiol 8. 2010:623-633.

7. Franci G, Falanga A, Galdiero S, Palomba L, Rai M, Morelli M, Galdiero M: Silver nanoparticles as potential antibacterial agents. J Mol . 2015:88568874
8. Ramasamy M, Lee J: Recent Nanotechnology Approaches for Prevention and Treatment of Biofilm-Associated Infections on Medical Devices. Biomed Res Int. 2016; 2016:1851242.

9. Kushwaha A, Singh VK, Bhartariya J, Singh P: Isolation and identification of $\mathrm{E}$. coli bacteria for the synthesis of silver nanoparticles: characterization of the particles and study of antibacterial activity. 2015;5(1):65-70.

10. Liao C, Li Y, Tjong SC: Bactericidal and cytotoxic properties of silver nanoparticles, International Journal of Molecular Sciences. 2019 doi: 10.3390/ijms20020449

11. Christensen GD, Simpson WA, Younger JJ, Baddour LM, Barrett FF, Melton DM, et al: Adherence of coagulase-negative staphylococci to plastic tissue culture plates: a quantitative model for the adherence of staphylococci to medical devices. Journal of clinical microbiology. 1985. 22(6): 9961006

12. O'Toole GA: Microtiter dish biofilm formation assay. Journal of Visualized Experiments. 2011. (47): e2437.

13. Namasivayam SKR, Roy EA: Enhanced antibiofilm activity of chitosan stabilized chemogenic silver nanoparticles against Escherichia coli. Int J Sci Res. 2013:59

14. Abdel Rahim KA, Mohamed AM: Bactericidal and antibiotic synergistic effect of nanosilver against methicillin resistant Staphylococcus aureus, Jundishapur J Microbiol. 2015; 8: e25867-e25873.

15. Niveditha S, Pramodhini S, Umadevi S, Kumar S, Stephen S. The isolation and the biofilm formation of uropathogens in the patients with catheter associated urinary tract infections (UTIs). Journal of clinical and diagnostic research: JCDR. 2012, 6(9), 1478.

16. Letica-Kriegel AS, Salmasian H, Vawdrey DK, Youngerman BE, Green RA, Furuya EY, ... \& Perotte R. Identifying the risk factors for catheterassociated urinary tract infections: a large crosssectional study of six hospitals. BMJ open. 2019, 9(2), e022137.

17. Sabir, N, Ikram, A, Zaman, G, Satti, L, Gardezi, A, Ahmed, A, \& Ahmed, P. Bacterial biofilm-based catheter-associated urinary tract infections: Causative pathogens and antibiotic resistance. American journal of infection control. 2017, 45(10), 1101-1105.

18. Lewis SS, Knelson LP, Moehring RW, Chen LF, Sexton DJ, Anderson DJ. Comparison of NonIntensive Care Unit (ICU) versus ICU Rates of Catheter-Associated Urinary Tract Infection in Community Hospitals. Infection Control \& Hospital Epidemiology. 2013, 34(7), 744-747. 
19. Hsiao CY, Lin HL, Lin YK, Chen CW, Cheng YC, Lee WC, Wu TC. Urinary tract infection in patients with chronic kidney disease. Turkish journal of medical sciences. 2014, 44(1), 145-149.

20. Tabasi M, Karam MRA, Habibi M, Yekaninejad MS, Bouzari S: Phenotypic assays to determine virulence factors of uropathogenic Escherichia coli (UPEC) isolates and their correlation with antibiotic References 184 resistance pattern. Osong public health and research perspectives. 2015. 6(4): 261 268.

21. Byarugaba DK: Antimicrobial resistance in developing countries and responsible risk factors. International journal of antimicrobial agents. 2004. 24(2): 105-110.

22. Elsayed Gawad W, Mohamed Helmy O, Mostafa Tawakkol W, Mohamed Hashem A. Antimicrobial resistance, biofilm formation, and phylogenetic grouping of uropathogenic Escherichia coli isolates in Egypt: The role of efflux pump-mediated resistance. Jundishapur Journal of Microbiology. 2018, 11(2).

23. Hassan A, Usman J, Kaleem F, Omair M, Khalid A, Iqbal M. Evaluation of different detection methods of biofilm formation in the clinical isolates. Brazilian journal of infectious Diseases. 2011, 15(4), 305-311.

24. Maharjan G, Khadka P, Siddhi Shilpakar G, Chapagain G, Dhungana GR. Catheter-associated urinary tract infection and obstinate biofilm producers. Canadian Journal of Infectious Diseases and Medical Microbiology. 2018.

25. Costa AF, Silva LDC, Amaral AC. Farnesol: An approach on biofilms and nanotechnology. Medical Mycology. 2021. 